\title{
FACTORES PSICOLÓGICOS DE INSUCESSO NA RESOLUÇÃO DE PROBLEMAS DE FÍSICA: UMA AMOSTRA SIGNIFICATIVA
}

\author{
NETO, A.J. \\ Universidade de Évora, 7000 Évora, Portugal
}

\section{SUMMARY}

A study of the influence of some pertinent psychological factors on physics problem-solving performance is attempted. The special cases of conceptual, verbal, and procedural information and cognitive development are analysed.

\section{INTRODUÇĀO}

Existe algum consenso na literatura da especialidade em admitir que um problema é uma questão para a qual não se conbece, à partida, solução, nem se sabe, sequer, se ela existirá (Garrett 1987). Ora, é indiscutível que a maior parte dos «problemas» utilizados pela Física escolar não obedecem àquele requisito. Trata-se, em geral, de exercícios de aplicação mecânica e rotineira para os quais o aluno sabe, antecipadamente, que existe solução (geralmente única) e que, além disso, apenas exigem que ele seja capaz de relembrar processos de resolução anteriormente memorizados (Gil Pérez e Martínez 'Torregrosa 1983, Valente et al. 1989, Garrett et al, 1989).

É inegável que tais exercícios têm a sua utilidade didáctica. Os procedimentos algorítmicos que lhes são inerentes constituem pré-requisitos importantes para a resolução de probiemas verdadeiros (Gagné 1985). Contuda, a didáctica das ciências não pode estar confinnada à utilização exclusiva de algoritmos desse tipo, sob pena de o desenvolvimento critico e criativo do aluno ficar decisivamente comprometido. Torna-se, assim, necessário o recurso à abordagem de problemas autênticos, abordagem essa cuja complexidade exige o contributo de uma diversidade de factores cognitivos, agrupados, por Gagné (1985), em três categorias de informação:

-Informação Conceptual: relacionada com o conhecimento de conceitos, por sua vez associados em regras básicas tais como leis, princípios ou algoritmos.
-Informaçäo Verbal: referente ao conhecimento de factos (informação factual) ou ao domínio da linguagem (conhecimento linguístico).

-Informação Processual: relativa ao conhecimento de estratégias cognitivas e metacognitivas as quais incluem os processos de armazenar e processar informação.

A amostra significativa que iremos adoptar, e à qual nos referimos no título deste trabalho, vai ser constituída por quatro componentes básicos: pelos factores associados a cada um daqueles três pré-requisitos e pelo factor desenvolvimento cognitivo, até aqui ainda não explicitado.

\section{A INFORMAÇÃO CONCEPTUAL}

A Física conjuga a especificidade conceptual e metodológica que the é propria corn a especificidade conceptual, estrutural e lógica que caracteriza a Matemática. Não admira, por isso, que a Matemática passe por ser a grande responsável pelo fracasso na resolução de problemas de Física, particularmente nos de ordem quantitativa.

A investigação (Gamble 1986, Jung 1985) tern provado, contudo, que grande parte das dificuidades dos aiunos face a esses problemas tem mais a ver com bloqueamentos no raciocínio qualitativo do que a incapacidades de ordem matemática. 
Os bloqueamentos no raciocínio qualitativo («raciocínio físicon) devem-se não so a questões relacionadas com a criação e execução de estratégias, mas também a desajustamentos conceptuais. Esses desajustamentos derivam do conflito que muitas vezes se estabelece entre os conceitos formais subjacentes ao problema e as concepç̃es alternativas próprias dos alunos (Driver 1983, Gilbert e Watts 1983, di Sessa 1987, Osborne e Freyberg 1987).

A aprendizagem dos tópicos de ciência formal faz-se a partir dessas concepçōes e é por elas fortemente condicionada. Poder-se-á, assim, afirmar, com Ausubel, que «o factor isolado que mais decisivamente influencia a aprendizagem é aquilo que o aluno já sabe» (Ausubel et al. 1978).

A ađopção do referencial científico escolar não é tarefa fácil. Fla exige, muitas vezes, o abandonoou a reformulação de concepçóes assimiladas e estruturadas ao longo de anos e profundamente incorporadas nas estruturas cognitivas do aluno. Exige, no fundo, aquilo que muitos autores designam por mudança conceptual (Posner et al. 1982, West e Pines 1985) a qual só é possível mediante a utilização de metođologias distintas da «metodologia da superficialidade» quase sempre dominante (Gil Pérez e Martínez 'Torregrosa 1983, Gil Pérez e Carrascosa $1985)$.

As consideraçōes anteriores aplicamse com particular acuidade à Física. De facto, é na Física, especialmente na Mecânica, que o conflito conceptual mais se faz sentir. Ao abordar problemas de Física o aluno é levado a ter de optar entre duas formas distintas de «ver» o mundo: uma concreta, intuitiva e de senso-comum (visâo pré-galileana) e outra abstracta e formal (visão cicntífica «oficial»). E evidente que se o aluno abordar o problema (suposto um problema «oficial») segundo uma perspectiva pré-galileana fracassará seguramente na sua resolução.

\section{INFORMAÇÃO VERBAL: O CASO PARTI- CULAR DA IINGUAGEM}

A informação verbal incorpora duas formas essenciais de conhecimento: o conhecimento factual e a linguagem (conhecimento linguístico).

O conhecimento factual exerce uma função determinante na resolução de problemas, na medida em que, complementando o conhecimento conceptual, é essencial para a compreensão e descodificação da natureza do problema.

O papel da linguagem é, contudo, mais profundo. A sua infiuência e relevância para o desenvolvimento cognitivo foi salientada por psicólogos como Piaget e Vygotski. Mas, enquanto que Piaget faz sobretudo ressaltar a importância da linguagem como veículo de comunicação entre a criança e o seu meio (função externa da linguagem), Vygotski vai mais longe. Este autor afirma que na fase inicial do desenvolvimento predomina, de facto, a vertente comunicativa da linguagem; nessa fase a linguagem é essencialmente interpessoal. Mas, o momento crucial do desenvolvimento intelectual da criancat ocorre a partir da altura em que, para além da sua funçăo interpessoal, a linguagem passa a desempenhar, também, uma função intrapessoal. t́ cssa internalização da linguagem que permite agora à criança realizar o pensamento elaborado e abstracto. Com ela, «o pensamento torna-se verbal c a linguagem racional» (Vygotski 1979, p.192).

A linguagem acaba, assim, por influenciar decisivamente a actividade de resolver problemas, na medida em que esta actividade pressupöe o recurso a formas elaboradas e abstractas de pensamento, ou seja, segundo Vygotski, a «funções psicológicas superiores».

No que diz respeito aos aspectos externos da linguagem, é na Física que, mais uma vez, essas questôes assumem especial pertinência. Com efeito, o código de linguagem da Física é, inevitavelmente, caracterizado pela predominância de termos técnicos e de conceitos abstractos, a cujo significado o aluno não consegue muitas vezes ter fácil acesso. Por outro lado, essa linguagem é apoiada numa lógica matemática fortemente estruturada e de natureza proposicional, bastante diferente da lógica simples c linear a que os alunos, sobretudo os mais novos, sãto mais facilmente permeáveis.

É por isso provável que, perante um problema de Física, o aluno não o consiga resolver deviđo à dificuldade em transpor o primeiro obstáculo, representado pela necessidade imperiosa de, através da descodificaçãodoenunciado, compreender qual é, no fundo, a «verdadeira natureza do problema» (Hayes 1976).

\section{A INFORMACÃO PROCESSUAL: ESTRA- TEGIAS COGNITIVAS}

As cstratégias cognitivas constituem planos de acção mental que orientam e controlam a utilização dos outros tipos de informação. Greeno (1980) reafirma a importância dessas estratégias, ao considerar que toda a actividade de resolução de problemas tem por base dois tipos de conhecimento: o conhecimento de estratégias cognitivas (conhecimento processual) e o conhecimento de conceitos (conhecimento conceptual). Concede grande ênfase à interrelação entre esses dois tipos de conhecimento na resolução de problemas. Nisso se distingue das correntes que poem a tónica no conhecimento conceptual -p.e., a tradição ausubeliana- ou das que sobrevalorizam a importância de estratégias gerais -p.c.,o movimento heurístico, representado pelo seu fundador Polya (1975).

À parte a controversia referente a qual dos conhecimentos tem influência mais determinante na resolução de problemas, é indiscutível que a escola não deve limitirse a transmitir informação conceptual (e factual). Terá também de se preocupar com o desenvolvimento do conhecimento processual do aluno, ou seja, terá de "Ensinar o Aluno a Pensar» (Valente et al. 1987). Para isso, é necessário que a escola seja capaz de: 
- Criar condições que possibilitem ao aluno a oportunidade de pôr em prática as operações cognitivas que já consegue realizar.

-Ensinar directamente aqueles processos intelectuais que, não sendo inerentes á matriz cognitiva do aluno médio, sejam, contudo, ensináveis (casos dos processos associados ao pensamento crítico e ao pensamento criativo).

-Fnsinar o aluno a tomar consciência dos seus próprios processos de pensamento, ou seja, levá-lo a desenvolver estratégias de tipo motacognitivo, através das quais ele possa controlar, gerir e avaliar toda a sua actividade intelectual (Costa 1985).

\section{AS LIMITAÇŌES DA MEMÓRIA OPE- RATIVA}

A importância e o cfeito da informação (conceptual, verbal ou processual) na resolução de problemas :êm sido repetidamente estudados pelos investigadores que adoptam como paradigma o modelo do processamento de informaçāo (Newell e Simon 1972, Gagné 1985). Yiste modelo atribui à memória a função central no que diz respeito à aprendizagem e à resolução de problemas. A memória, segundo o modelo, inclui duas vertertes fundamentais: a memória a curto prazo responsável pela função dupla de armazenar temporariamente informação e de a processar; a memória a longo prazo, responsável pelo armazenamento (retenção) de todo o conhecimento previamente aprendido, o qual nela se organiza segurto estruturas habitualmente designadas por «esquemas».

A memória a curto prazo (memória operativa) tem una capacidade de processamento simultâneo limitada a, aproximadamente, sete unidađes de memória (Hayes 1976). Cada unidade de memória corresponde a um conjunto organizado de itens de informação ao quual o procesiador, devido a aprendizagens prévias, é capaz de atribuir um significado global e único. Segundo esta corrente, cste condicionamento da memória operativa é o factor que justifica a existência da maior parte dos bloqueamentos cognitivos face à resolução de problemas.

Quanto major for o número de unidades de memória presentes na memória operativa menor é a probabilidade de o problema ser resolvido com sucesso. Torna-se por isso necessário reduzir o número de unidades de memória a que se tem de recorrer. Essa redução está dependente do grau de organização e estruturação do conhecimento presente na memória a longo prazo (Larkin e Reif 1979, Pankratius 1990, Neto e Almeida 1990).

Uma grande parte dos problemas de Física exige o recurso a doses elevadas de informação, quer ela provenha do exterior ou seja recordada. A primeira dificuldade reside, assim, no número de unidades de memória que o aluno terá de utilizar. Mas, outra dificuidade surge, a qual está relacionada com a dimensão e interligação dessas unidades de memória. Em virtude da Física ser uma disciplina fortemente estruturada, os seus conceitos organizam-se segundo redes complexas com elos interdepen-dentes e ramificados. Ao formar as unidades de memória de que necessita, o aluno terá de ser capaz de reconstituir algumas dessas redes o que, em muitos casos, é tarefa problemática. Para que o não fosse, a aprendizagem dos tópicos de Física teria de ser verdadeiramente significativa e não ser realizada, como tradicionalmente acontece, por simples justaposição e acumulação de conhecimentos mais ou menos desconexos.

\section{O DESENVOLVIMENTO COGNITIVO}

A aprendizagem de Física, sobretudo a partir do ensino secundário, requer dos alunos o domínio de algumas das mais importantes operações formais piagetianas. Muitos autores, principalmente os que trabalham na base do paradigma de Piaget, têm identificado correlações positivas entre a capacidade efectiva de realizar as operaçōes formais e o grau de sucesso na resolução de problemas de Física. O domínio pelo aluno dessas operações constituiria, assim, mais um pré-requisito crucial para a resolução desses problemas.

No entanto, estudos realizados em países tão diversos como os Estados Unidos (Chiappetta 1976), Inglaterra (Shayer e Adey 1981), Portugal (Sequeira 1981) ou Jordânia (Billeh e Khatili 1982) permitiram concIuir que uma parte significativa dos adolescentes (por vezes mais de 50\%) não consegue operar satisfatoriamente a um nivel formal. Significa isto que o ensino da Písica é fortemente problemático no que diz respeito ao seu impacte positivo na aprendizagem desses alunos.

\section{CONSIDERAÇÓES FINAIS}

Procurando identificar alguns factores psicologicos que, de algum modo, possam infuenciar a actividade de resolução problemas de Física, concluímos serem pertinentes os seguintes: a base conceptual do aluno quer ela se refira a conhecimentos específicos, conhecimentos matemáticos ou conhecimentos alternativos, $a$ informação verbal que o aluno possui, nomeadamente a que se relaciona com a linguagem; as estratégias cognitivas e metacognitivas que o aluno conhece; as limitaçôes da sua memória operativa e a forma como os conhecimentos estão organizados na memória a longo prazo; o seu desenvolvimento cognitivo, particularmente no que diz respeito à realização das operações formais.

É cvidente que outros factores poderíamos ter mencionado (Garrett 1986). É o caso, por exemplo, dos que se relacionam com os estilos cognitivos e motivacionais próprios de cada aluno ou dos que estão associados a variáveis de natureza sócio-afectiva. 'Todavia, a amostra por nós analisada, parece-nos suficientemente significativa para que possamos concluir que a resoluçẫo de problemas de Física, ao invés de ser encarada como uma actividade rotincira e monolítica, deve, antes, ser vista 
como uma tarefa complexa e multidimensional. Fssa complexidade exige o recurso a uma didáctica oposta à «didáctica da superficialidade» habitualmente adoptada. Ela requer, assim (Gil Péréz e Carrascosa 1985), a

\section{NOTA}

Foi apresentada uma primeira versão deste artigo no I Seminário «A Componente de Psicologia na Formação de Professores», Évoria, Setembro de 1990.

\section{REFERÊNCIAS BIHLIOGRÁFICAS}

AUSUBEL, D.P., NOVAK, J.D. C HANESIAN, H., 1978. Educutional Psychology: A Cognitive View $\left(2^{a}\right.$ ed.). (New York: Holt, Reinhart and Winston).

BII LEH, V.e KHALILI, K., 1982. Cognitive development and comprehension of physics concepts, European Journal of Science Education, 4 (1), pp. 95-104.

CHIAPPETTA, E.L., 1976. A review of Piagetian studies relevant to science instruction at secondary and college level, Science Education, 60 (21), pp. 253-261.

COSTA, A.L., 1985. Teaching for, of, and about thinking, en A. 1. Costa (ed.), Developing Minds: a Resource Book for Teaching Thinking. (Alexandria, VA: ASCD Fdition).

DI SESSA, A.A., 1987. The third revolution in computers and education, Joumal of Research in Science Teaching, 24(4), pp. 343-367.

DRIVER, R., e ERICKSON, G., 1983. Theorics in action: some theoretical and empirical issues in the study of conceptual frameworks in science, Studies in Science Education, 10,pp. $37-60$.

GAGNE, R.M., 1985. The Conditions of Learning (4' edição). (Tóquio: Holt Saunders Japan).

GAMBIE, R., 1986. Simple equations in physics, European Joumal of Science Education, 1(1), pp. 27-37.

GARRY'TT, R. M., SAITERLY, D., GIL PEREZ, D. e MARTINHZ TORREGROSA, J. 1989. Turning exercises into problems: an experimental study with teachers in training, International Joumal of Science Education, 9(2), pp. 125137.

GARRETT, R.M., 1986. Problem-solving in science education, Studies in Science Education, 13, pp. 70-95. utilização de estratégias capazes de asscgurarem a $m$ itdança metodológica dos professores sem a qual a desejada mudança conceptual dos alunos dificilmente ocorrerá.
GARRETT, R. M. 1987. Issues in science education: problem solving, creativity and originality, International Joumal of Science Education, 9(2), pp. 125-137.

GL PEREZ, D. e CARRASCOSA, J. 1985. Science learning as a methodologicat and conceptual change, European Journal of Science Education, 7(3), pp. 231-236.

GIL PÉREZ, D. e MARTÍNFZ TORRFGROSA, J., 1983. A model for problem solving in accordance with scientific methodology, European Journal of Science Education, 54(4), pp. $448-457$.

GILBERT, J.K. e WATTS, D.M., 1983. Conceptions, misconceptions and alternative conceptions: changing perspectives in science education, Studies in Science Kiducation, 10, pp. 61-98.

GREENO, J.G., 1980. Trends in the therry of knowledge for problem solving, in D.T.'Tuma eF. Reif (eds.), Problem Solving and Education. (Hillsdale, $\mathrm{N} \mathrm{J}$ : Latwrence Erlbaum Assicialtes).

HAYFS, J.R, 1976. The Complete Problem Soluser. (Hillsdale, NJ: Lawrence Èribaum Associates).

IJNG, W., 1985. Uses of cognitive science to science education. Paper presented to the A'TEE Symposium on the implications of cognitive science for the education of science teachers, Kiel, August.

LARKIN, J.H. e RYIF, Fi, 1979. Understanding and teaching problem-solving in physics, European Journal of Sctence Education, 1(2), pp. 191-203.

NETO, A.J. e ALMEIDA, M., 1990. Conhecimento, lógicas de organizaçāo e rendimento escolar, O Professor, 12 (3" Sćrie), pp. 40-51. 
NEWELL, A. e SIMON, H. A. 1972. Human Problem Soiving. (Englewood Cliffs: Prentice-Hall).

OSBORNE, R. e FRFYBERG, P., 1985. Learning in ScienceThe Implications of Children's Science. (Auckland: Heinemann).

PANKRATIUS, W.J., 1990. Building an organized knowledge base: concept mapping and achievement in secondary school physics, Journal of Research in Science Teaching, 27(4), pp. $31.5-333$.

- POLYA, G. 1973. How to Solve it ( 2 edição). (New Jersey: Princeton University Press).

POSNER, G.I. et al., 1982. Accomodation of a scientific conception: toward a theory of conceptual change, Science Education, 66(2), pp. 211-217.

SEQUEIRA, M., 1981. Padrōes de raciocínio em alunos portugueses: implicaçōes para o curriculum e ensino das ciências na escola secundária, Aprendizagem e Desenvolvimento, 1(3), pp. 39-50.

SHAYER, M. e ADEY, P., 1983. Towards a Science of Science Teaching. (London: Heinemann).

VALENTE, M.O., NETO, A.J. e VALENTE, M., 1989. Resolução de problemas em Física: necessidade de uma ruptura com a didáctica tradicional, Gazeta de Fisica, $12(3)$, pp. $70-$ 78 .

VALENTE, M.O. et al,, 1987. Aprender a Pensar. Lisboa: Projecto DIANOHA, Departamento de Educação da Faculdade de Ciências da Unjversidade de Lisboa.

VYGOTSKI, L.S., 1979. El Desarollo de los Processos Psicologicos Superiores. (Barcelona: Editorial Crítica).

WEST, L. C PINES, A., 1985. Cognitive Structure and Conceptual Change. (Orlando: Academic Press). 


\section{FACTORES PSICOIÓGICOS DE FRACASO EN LA RESOLUCION DH, PROBLEMAS DE FISICA: UNA MUESTRA SIGNIFICATIVA}

\section{RESLMEN}

\section{Introducción}

Aunque existe consenso en la literatura especializada en definir problema como una cuestión para la que no se conoce de antemano ni la solución ni si ésa existe, la mayoría de los probiemas de Física propuestos no responden a tal requisito, tratándose de ejercicios de aplicación mecánica. Tales cjercicios tjenen su utilidad, pues los procedimientos algorítmicos que comporta su solución son prerrequisitos importantes para la resolución de verdaderos problemas.

Resulta imprescindible abordar la resolución de problemas. Diversos factores cognitivos que contribuyen a ello son agrupados por Gagné en tres catcgorías de información:

- Información conceptual, relacionada con el conocimicnto de conceptos, leyes, principios y teorias.

- Información verbal, referida al conocimineto de hechos o a) dominio del lenguaje.

- Infonnación procesual, relativa al conocimiento de estrategias cognitivas o metacognitivas, incluyendo los procesos de almacenamiento y procesamiento de la información.

Estos tres componentes, funto al factor del desarrollo cognitivo, forman lo que llamamos una muestra significativa.

\section{Información conceptual}

La Física conjuga la especif́cidad conceptual y metodológica propia con la especificidad conceptual,estructural y lógica de las Matemáticas. Se considera al aparato matemático responsable del fracaso en la resolución de problemas de Física. La investigación, sin embargo, muestra que la mayor parte de estas dificultades tienen relación con bloqueos en el razonamiento cualitativo. Estos bloqueos se deben a cuestiones relacionadas con la creación y aplicación de estrategias y a desajustes conceptuales derivados, en muchos casos, de las concepciones alternativas de los alumnos

\section{Información verbal.}

Incorpora dos fornas esenciales de conocimiento: el factual y el del lenguaje.

El papel del lenguaje es más profundio. Vygotski va más allá que Piaget en sus propuestas acerca del papel del lenguaje en cl desarrolto. Para este autor, en la fase inicial del desarrollo predomina la vertiente comunicativa $\mathrm{e}$ interpersonal del lenguaje. Fil momento crucial del desarrollo intelectual de los nifios se produce al desempañar el lenguaje una función intrapersonal que les permite llevar a cabo el pensamiento claborado y abstracto. «Se verbaliza el pensamiento y se racionatiza el lenguaje». El lenguaje influye, así, en la resolución de problemas. El código linguístico de la Física se caracteriza por el predominio de términos técnicos, apoyados por una lógica matemática, fuertemente estructurada, de naturaleza proposicional.

Es probable, que el alumno no consiga tesoiver el problema debido a que la dificultad en la comunicación del enunciado le impida comprender la verdadera naturaleza del problema.

\section{Información personal: estrategias cognitivas}

Las estrategias cognitivas constituyen planes de acción mental que orientan y controlan la utilización de otros tipos de información.Según Greeno toda actividad de resolución de problemas se basa en dos tipos de conocimiento: el de estrategias cognitivas y el de conceptos. Ambos están fucrtemente interrelacionados en la resolución de problemas. Otros autores ponen más el acento en uno a otro conocimiento.

De todos modos, es indiscutible que la ensef̂anza no debe Iimitarse a la transmisión de información conceptual y factual, y debe preocuparse dei desarrotlo del conocimiento procesual. Para ello debe:

- Crear condiciones que posibiliten que el alumno ponga en práctica las operaciones cognitivas que ya consigue realizar.

- Enseñar directamente aquellos procesos intelectuales que, siendo interesantes a la «matriz» cognitiva de algún alumno medio, sean enseñables.

-Enseñar al alumno a tomar conciencia de sus propios procesos de pensamiento y llevar lo a desarrollar estrategias de tipo metacognitivo.

\section{Las limitaciones de la memoria operativa.}

La importancia del efecto de la información ha sido estudiada por los investigadores del modelo de procesamiento de la información. Distinguen entre memoria a corto y a largo plazo.

La memoria a corto plazo o memoria operativa tiene una capacidad de procesamiento limitada a siete unidades cada una de las cuales puede considerarse un bloque de información organizada. Este factor justifica la mayor parte de los bloqueos cognitivos en ta resolución de problemas.

Una gran parte de los problemas de Física exige grandes cantidades de información lo que supone que el alumno utilizará muchas unidades de memoria. Además, al ser la Física una disciplina fuertemente estructurada tiene los conceptos organizados en complejas redes con muchas interdependencias lo que, en algunos casos, exige su reconstrucción por parte del alumno, y esto es difícil y requiere un aprendizaje verdaderamente significativo.

\section{El desarrollo cognitivo}

El aprendizaje de la Física, sobre todo a partir de secundaria requiere el dominio de las operaciones formales piagetianas más importantes por parte del alumno. Muchos autores han establecido correlaciones entre el dominio formal y el nivel de éxito en la resolución de problemas. El dominio de estas operaciones sería un prerrequisito crucial para la resolución de problemas. Sin embargo, varios estudios indican que una parte importante de los adolescentes (el $50 \%$ o más) no alcanza satisfactoriamente el nivel formal, con la dificultak que esto supone para la enseñanza de la Física.

\section{Consideraciones finales}

Algunos factores psicologicos que influyen en la resolución de problemas de Fisica son: la base conceptual, la información verbal, las estrategias cognitivas, las limitaciones de la memoria operativa y el desarrollo cognitivo. 